The Egyptian Journal of Hospital Medicine (January 2021) Vol. 82 (3), Page 416-425

\title{
Association of Circulatory Long Non-Coding RNA AFAP1-AS1 with Risk and Progression of Hepatocellular Carcinoma in Egyptian Patients with Chronic Hepatitis C Virus Infection
}

\author{
Nearmeen M. Rashad*1, Sherweet M. Ahmed ${ }^{2}$, Marwa H.S. Hussien ${ }^{3}$, Marwa G. Amer ${ }^{4}$, Neveen F. Ibrahim ${ }^{1}$ \\ Departments of ${ }^{1}$ Internal Medicine, ${ }^{2}$ Tropical Medicine, ${ }^{3}$ Medical Biochemistry and \\ ${ }^{4}$ Clinical Pathology, Faculty of Medicine, Zagazig University - Zagazig, Egypt \\ *Corresponding author: Nearmeen M. Rashad, Tel.: +20 1224248642, E-mail: nrashad78@yahoo.com \& n.rashad@zu.edu.eg.
}

\begin{abstract}
Background: Hepatocellular carcinoma $(\mathrm{HCC})$ is one of the most frequent malignant tumors worldwide. Long noncoding RNA (lncRNA) contribute to extensive biological processes and play oncogene or tumor suppressor roles in several diseases.

Objective: To evaluate the expression levels of lncRNA AFAP1-AS1 in Egyptian patients with chronic hepatitis c virus infection (HCV) and to assess its relations with clinicopathological features of HCC.

Patients and Methods: 60 chronic hepatitis $\mathrm{C}(\mathrm{CHC})$ patients in addition to 40 healthy subjects as a control group were enrolled in this study. CHC patients were divided into three groups, group I comprised 33 patients with chronic hepatitis $\mathrm{C}(\mathrm{CHC})$; group II comprised 15 patients with cirrhosis and 12 patients with HCC. lncRNA AFAP1-AS1 relative expression level was determined by RT-PCR.

Results: IncRNA AFAP1-AS1 relative expression level was upregulated in CHC groups (4.28 \pm 2.69$)$ compared to controls $[(2.56 \pm 1.35), \mathrm{P}<0.001 *]$. Additionally, there was a significant difference between case groups as the highest relative expression level was in HCC patients $(5.3 \pm 2.28)$ compared to cirrhosis $(4.28 \pm 3.69)$ and CHC $(3.93 \pm 0.652)$. The lncRNA AFAP1-AS1 relative expression level was significantly positively correlated with clinicopathological features of HCC.

Conclusions: lncRNA AFAP1-AS1 relative expression level was upregulated in CHC patients in particular patients with cirrhosis and HCC. Thus, circulatory lncRNA AFAP1-AS1 may be able to serve as a promising non-invasive diagnostic marker for cirrhosis and HCC.
\end{abstract}

Keywords: lncRNA, Chronic hepatitis C, Cirrhosis, AFAP1-AS1, HCC.

\section{INTRODUCTION}

Hepatocellular carcinoma (HCC) is one of the most frequent malignant tumors worldwide. Chronic hepatitis $\mathrm{C}(\mathrm{HCV})$ infection is the third leading cause of HCC and accounts for about one-third of total incidence rates and one-fifth of HCC-related deaths (1). HCV increases the risk of HCC development by nearly 17 -fold compared to healthy individuals ${ }^{(2)}$. The risk of developing $\mathrm{HCC}$ in patients with $\mathrm{HCV}$ infection increases in proportion to the degree of hepatic fibrosis. In fact, most cases of HCV-related HCC occur during established cirrhosis, suggesting that cirrhosis-mediated carcinogenesis may play a primary role in the development of $\mathrm{HCC}^{(3)}$.

Long non-coding RNA (LncRNAs) account for a large portion of the non-coding transcripts. They include more than 200 nucleotides and have no or restricted protein-coding potential ${ }^{(4)}$. LncRNAs can regulate gene expression and the synthesis of protein in different ways. Numerous previous studies concluded that long noncoding RNAs play an important role in regulating many biological processes including gene expression, cell cycle regulation, and cellular differentiation (5). Hence, pathogenesis and pathophysiology such as cancers, cardiovascular disorders, and metabolic disorders. This

relation reveals that IncRNAs could be used for therapeutic targets or biomarkers for diseases ${ }^{\left({ }^{(}\right)}$. lncRNA actin filament associated protein 1antisense RNA 1 (AFAP1-AS1) is associated with cellular proliferation, invasion, and metastasis and increased apoptosis in vivo ${ }^{(7)}$.

Many global studies have focused on the diagnosis and treatment of HCC. Nevertheless, its prognosis is still poor owing to a series of reasons, such as growing incidence, late diagnosis, persistent drug resistance, and repeated recurrence. Thus, it is urgent to discover novel and valuable diagnostic and prognostic biomarkers for HCC the present study aimed to evaluate the expression levels of lncRNA AFAP1-AS1 in Egyptian patients with $\mathrm{HCV}$ and to assess its relations with clinicopathological features of HCC.

\section{SUBJECTS AND METHODS}

This case-control study was conducted on 60 adult

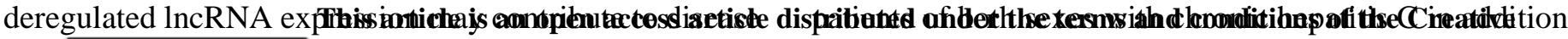


to 40 healthy control individuals,' age, ethnicity, sex, and smoking matched to the cases. Patients were divided into three groups; group I comprised 33 patients with chronic hepatitis $\mathrm{C}$ (CHC), group II comprised 15 patients with cirrhosis, group III included 12 patients with HCC.

\section{Ethical and patients' approval:}

The approval from the Ethics Committees, Faculty of Medicine, Zagazig University was taken. All participants' signed informed written consents. All participants underwent complete history taking and thorough clinical examination. All patients were subjected to abdominal ultrasonography using probe $3.5 \mathrm{MHZ}$ of TDI Philips machine.

Liver biopsies were obtained using an automated gun device and under complete aseptic precautions. Pathological examination of the liver biopsy was performed by an experienced pathologist who was blinded for patients' data. HCC was diagnosed according to the American Association for the Study of Liver Diseases practice guidelines. Clinical staging of HCC was evaluated cording to the Barcelona Clinic Liver Cancer staging classification ${ }^{(8)}$ and Child-Pugh classification ${ }^{(9)}$. Clinicopathological features of HCC cases including tumor number, size, site, presence of metastasis, and portal vein thrombosis were obtained at the time of blood collection.

Patients with chronic HBV infection or any other cause for chronic hepatitis other than HCV were excluded from the protocol. No patient had heart failure, renal failure, active bacterial infections, and evidence of other endocrine disorders or received hormone replacement therapy. Patients with other causes of chronic liver disease apart from HCV infection were excluded from the study.

\section{Laboratory tests:}

Blood samples were drawn from all subjects after an overnight fast. We divided the blood sample into 2 portions: $1 \mathrm{ml}$ of whole blood was collected into tubes containing EDTA for miRNA extraction. Sera were separated immediately from the remaining third portion and stored at $-20{ }^{\circ} \mathrm{C}$ until analysis. Complete blood count (CBC), Prothrombin time (PT) and international normalized ratio (INR) were performed for all patients. We used full automated Cobas 8000 c702 (Roche diagnostic, Germany) for bilirubin, ALT, AST and creatinine. Electrochemiluminescence immunoassay on full automated Cobas 8000 e601 (Roche diagnostic, Germany) for alpha-fetoprotein \& viral markers HCV abs and HBV sAg

RNA isolation and quantitative real-time polymerase chain reaction (qRT-PCR)
Total RNA was isolated from tissues and cells using Trizol Reagent (Invitrogen) according to the manufacturer's protocol. RNA concentration and integrity were determined using the NanoDrop2000 spectrophotometer (Thermo Fisher Scientific, Waltham, MA, USA) and standard RNA gel electrophoresis. Real-time PCR was performed using SYBR ${ }^{\circ}$ Green (TaKaRa, Dalian, China) and the ABI Prism 7900 Sequence Detection System (Applied Biosystems, Foster City, CA, USA) according to the manufacturer's protocol. The primers used were as follows: AFAP1-AS1, forward 5' TCGCTCAATGGAGTGACGGCA-3' and reverse 5'CGGCTGAGACCGCTGAGAACTT-3'; GAPDH, forward 5'- CACCCACTCCTCCACCTTTG-3' and reverse 5'- CCACCACCCTGTTGCTGTAG-3'. The foldchange for AFAP1-AS1 was normalized to GAPDH.

\section{Statistical analysis}

Data analysis was performed using Statistical Package for the Social Sciences Software (SPSS Version 20, Chicago, Illinois). Data were expressed as mean \pm standard deviation (SD), or number (percentage) when appropriate. Comparisons between two groups were performed using chi-squared $\left(\mathrm{X}^{2}\right)$ test. A one-way analysis of variance (ANOVA) test was used to compare between more than 2 groups. The relationships of IncRNA AFAP1AS1 relative expression level with clinical and biochemical characteristics' were tested with Pearson correlation. Evaluation of independent factors correlated with lncRNA AFAP1-AS1 relative expression level in HCC group were investigated with linear regression test. Receiver operating characteristic analysis was performed to assess the potential diagnostic accuracy of lncRNA AFAP1-AS1 relative expression level, the area under the curve (AUC), and the cutoff values. We considered $\mathrm{P}$ significant at $\leq 0.05$ with a $95 \%$ confidence interval (CI).

\section{RESULTS}

Clinical and biochemical characteristics of the studied groups.

Our results observed that $72.5 \%$ of the control group from a rural area and $56.7 \%$ of the case group from a rural area. In addition, there were significant differences between both groups as regards prevalence of diabetes mellitus and family history of HCC. Also, we found significantly higher levels of INR, PT, ALT, AST, total bilirubin, direct bilirubin, WBC count, serum creatinine as well as alpha-fetoprotein in the case group compared to the control group. On the contrary, we detected a significantly lower level of albumin, hemoglobin, and platelet in CHC patients compared to healthy controls, ( $\mathrm{P}$ $<0.001 *)$ as shown in table (1). 
Table (1): Clinical and laboratory characteristics of the studied groups

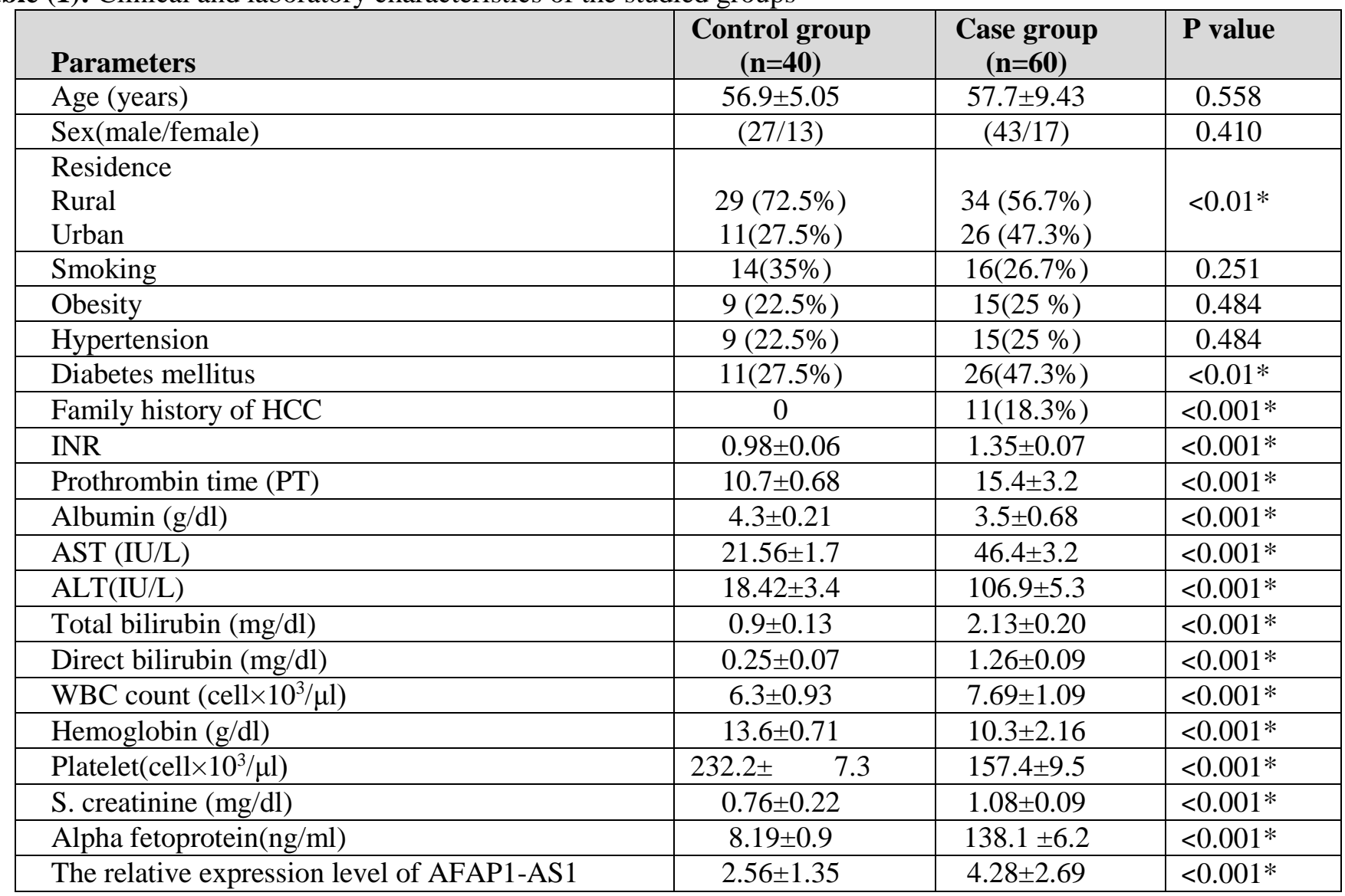

Clinical and biochemical characteristics of case groups

Our results revealed that there were significant differences between case groups as regards the prevalence of smoking and diabetes mellites. Additionally, there were significantly higher values of INR, PT, ALT, AST, total bilirubin, direct bilirubin, WBC count, serum creatinine as well as alpha-fetoprotein in HCC group compared to CHC and cirrhosis. On the other hand, we detected a significantly lower level of albumin, hemoglobin, and platelet in $\mathrm{CHC}$ patients compared to healthy controls [P $<0.001 *$ (Table 1$)]$ and $[\mathrm{P}<0.001 *$ (Table 2)].

The relative expression level of lncRNA AFAP1-AS1 in studied groups
This study was the first that investigated the relative expression level of lncRNA AFAP1-AS1 in Egyptian patients infected with HCV to find an early and non-invasive biomarker for HCC. There were significant high values in the case group $(4.28 \pm 2.69)$ compared to controls $(2.56 \pm 1.35)$ as shown in Table $(1)(\mathrm{P}<0.001 *)$. Additionally, there was a significant difference between case groups as the highest relative expression level was in HCC patients $(5.3 \pm 2.28)$ compared to cirrhosis $(4.28 \pm$ 3.69) and CHC (3.93 \pm 0.652$)$ as shown in table (2) (P $<0.001 *)$. 
Table (2): Clinical and laboratory characteristics of case groups

\begin{tabular}{|c|c|c|c|c|}
\hline Parameters & CHC, $(n=33)$ & Cirrhosis, $(n=15)$ & HCC, $(n=12)$ & P value \\
\hline Age (years) & $56.9 \pm 8.83$ & $55.7 \pm 13.20$ & $60.2 \pm 4.8$ & 0.319 \\
\hline Sex(male/female) & $(22 / 11)$ & $(8 / 7)$ & $(4 / 8)$ & 0.131 \\
\hline Residence & & & & \\
\hline Rural & $22(66.7 \%)$ & $8(53.3 \%)$ & $4(33.3 \%)$ & 0.131 \\
\hline Urban & $11(33.3 \%)$ & $7(46.7 \%)$ & $8(66.7 \%)$ & \\
\hline Smoking & $24(72.7 \%)$ & $8(53.3 \%)$ & $4(33.3 \%)^{\#, \delta}$ & $<0.05^{*}$ \\
\hline Obesity & $22(66.7 \%)$ & $7(46.7 \%)$ & $8(66.7 \%)$ & 0.131 \\
\hline Hypertension & $11(33.3 \%)$ & $8(53.3 \%)$ & $4(33.3 \%)$ & 0.131 \\
\hline Diabetes mellites & $24(72.7 \%)$ & $8(53.3 \%)$ & $4(33.3 \%)^{\#, \delta}$ & $<0.05^{*}$ \\
\hline Family history of HCC & $13(39.4 \%)$ & $7(46.7 \%)$ & $8(66.7 \%)$ & 0.268 \\
\hline INR & $0.88 \pm 0.06$ & $1.35 \pm 0.37^{\#}$ & $1.66 \pm 0.54^{\#, \delta}$ & $<0.001^{*}$ \\
\hline Prothrombin time (PT) & $11.6 \pm 0.98$ & $15.8 \pm 2.4^{\#}$ & $19.6 \pm 4.15^{\#, \delta}$ & $<0.001^{*}$ \\
\hline Albumin (g/dl) & $4.3 \pm 0.21$ & $3.5 \pm 0.68^{\#}$ & $2.75 \pm 0.57^{\#, \delta}$ & $<0.001^{*}$ \\
\hline AST (IU/L) & $21.56 \pm 1.7$ & $46.4 \pm 3.2^{\#}$ & $90.5 \pm 5.98^{\#, \delta}$ & $<0.001^{*}$ \\
\hline ALT(IU/L) & $95.3 \pm 3.4$ & $124.9 \pm 5.3^{\#}$ & $55.6 \pm 2.53^{\#, \delta}$ & $<0.001^{*}$ \\
\hline Total bilirubin $(\mathrm{mg} / \mathrm{dl})$ & $0.9 \pm 0.13$ & $2.13 \pm 0.20$ & $2.16 \pm 0.54$ & $<0.001^{*}$ \\
\hline Direct bilirubin $\mathrm{mg} / \mathrm{dl}$ ) & $0.25 \pm 0.07$ & $1.26 \pm 0.09$ & $1.26 \pm 0.13$ & $<0.001^{*}$ \\
\hline WBC count $\left(\right.$ cell $\left.\times 10^{3} / \mu \mathrm{l}\right)$ & $8.28 \pm 0.89$ & $7.09 \pm 1.88$ & $7.06 \pm 1.21^{\#}$ & $<0.001^{*}$ \\
\hline Hemoglobin (g/dl) & $13.6 \pm 0.71$ & $10.3 \pm 2.16$ & $9.42 \pm 2.09$ & $<0.001^{*}$ \\
\hline Platelet $\left(\right.$ cell $\left.\times 10^{3} / \mu \mathrm{l}\right)$ & $155.2 \pm 4.3$ & $140.4 \pm 4.5$ & $11.65 \pm \quad 4.2$ & $<0.001^{*}$ \\
\hline S. creatinine $(\mathrm{mg} / \mathrm{dl})$ & $0.96 \pm 0.25$ & $1.08 \pm 0.03^{\#}$ & $1.87 \pm 0.4^{\#, \delta}$ & $<0.001^{*}$ \\
\hline Alpha fetoprotein(ng/ml) & $11.56 \pm 4.7$ & $21.4 \pm 2.2^{\#}$ & $1895 \pm 5.98^{\#, \delta}$ & $<0.001^{*}$ \\
\hline $\begin{array}{l}\text { The relative expression level of } \\
\text { AFAP1-AS1 }\end{array}$ & $3.93 \pm 0.652$ & $4.28 \pm 0.69^{\#}$ & $5.3 \pm 2.28^{\#, \delta}$ & $<0.001^{*}$ \\
\hline
\end{tabular}

CHC, chronic hepatitis C; INR, international normalized ratio; ALT, alanine aminotransferase; AST, aspartate; aminotransferase; WBC, white blood cell count. $\quad$ "Significant $\mathrm{P}$ values $(\mathrm{P}<0.05)$ when compared with CHC group. ${ }^{\delta}$ Significant $\mathrm{P}$ values $(\mathrm{P}<0.05)$ when compared with cirrhosis group.

Concerning the relationship between the relative expression levels of AFAP1-AS1and clinicopathological characteristics in HCC patients, our results showed that about $83.3 \%$ of HCC patients were in stage III/IV, and $83.3 \%$ of patients had tumor size $>5 \mathrm{~cm}$. Interestingly, there were statistically significant differences between HCC patients regarding Child-Pugh grade, portal vein thrombosis, and number of tumor lesions, $\mathrm{P}<0.001^{*}$. However, there were non-significant differences between
HCC patients regarding lymph node metastasis and distant metastasis $\mathrm{P}>0.05$ (Table 3).

Regarding the relative expression level of AFAP1AS1 in correlation with clinicopathological characteristics in $\mathrm{HCC}$ patients, there were significantly higher levels in patients that had tumor size $>5 \mathrm{~cm}$, stage III/IV, ChildPugh grade $\mathrm{C}$, positive portal vein thrombosis and multiple tumor lesions $\left(\mathrm{P}<0.001^{*}\right)$ as shown in table (3). 
Table (3): The relationship between the relative expression level of AFAP1-AS1and clinicopathological characteristics in $\mathrm{HCC}$ patients

\begin{tabular}{|c|c|c|}
\hline & $\begin{array}{c}\text { HCC, }(\mathrm{n}=12) \\
\text { n }(\%)\end{array}$ & $\begin{array}{c}\text { The relative expression } \\
\text { level of AFAP1-AS1 }\end{array}$ \\
\hline \multicolumn{3}{|l|}{ Stage } \\
\hline Stage I/II & $2(16.7 \%)$ & $3.73 \pm 0.651$ \\
\hline Stage III/IV & $10(83.3 \%)$ & $5.55 \pm 1.32$ \\
\hline $\mathrm{P}$ value & $<0.01 *$ & $<0.001 *$ \\
\hline \multicolumn{3}{|l|}{ Tumor size } \\
\hline$<5 \mathrm{~cm}$ & $2(16.7 \%)$ & $3.13 \pm 0.563$ \\
\hline$>5 \mathrm{~cm}$ & $10(83.3 \%)$ & $6.93 \pm 1.112$ \\
\hline $\mathrm{P}$ value & $<0.01 *$ & $<0.001 *$ \\
\hline \multicolumn{3}{|c|}{ Lymph node metastasis } \\
\hline -Absent & $8(66.7 \%)$ & $5.11 \pm 0.212$ \\
\hline -Present & $4(33.3 \%)$ & $6.01 \pm 0.123$ \\
\hline $\mathrm{P}$ value & 0.248 & 0.426 \\
\hline \multicolumn{3}{|c|}{ Distant metastasis } \\
\hline -Absent & $9(75 \%)$ & $5.56 \pm 1.1$ \\
\hline -Present & $3(25 \%)$ & $6.01 \pm 0.98$ \\
\hline $\mathrm{P}$ value & 0.083 & 0.656 \\
\hline \multicolumn{3}{|c|}{ Child-Pugh grade } \\
\hline$-\mathrm{A}$ & $1(8.3 \%)$ & $3.23 \pm 0.22$ \\
\hline$-\mathrm{B}$ & $3(25 \%)$ & $5.83 \pm 1.6$ \\
\hline$-\mathrm{C}$ & $8(66.7 \%)$ & $6.12 \pm 1.7$ \\
\hline $\mathrm{P}$ value & $<0.05^{*}$ & $<0.001^{*}$ \\
\hline \multicolumn{3}{|c|}{ Portal vein thrombosis } \\
\hline -Negative & $9(75 \%)$ & $4.01 \pm 0.234$ \\
\hline -Positive & $3(25 \%)$ & $6.45 \pm 0.879$ \\
\hline $\mathrm{P}$ value & 0.083 & $<0.05^{*}$ \\
\hline \multicolumn{3}{|c|}{ Number of tumor lesions } \\
\hline -Single & $8(66.7 \%)$ & $4.13 \pm 0.6$ \\
\hline -Multiple & $4(33.3 \%)$ & $6.93 \pm 1.3$ \\
\hline $\mathrm{P}$ value & 0.248 & $<0.001^{*}$ \\
\hline \multicolumn{3}{|c|}{ Site of lesions } \\
\hline -Right lobe & $3(25 \%)$ & $5.6 \pm 1.53$ \\
\hline -Left lobe & $1(8.3 \%)$ & $5.1 \pm 1.75$ \\
\hline -Both & $8(66.7 \%)$ & $6.8 \pm 0.95$ \\
\hline $\mathrm{P}$ value & 0.039 & 0.940 \\
\hline
\end{tabular}

* Significant $\mathrm{P}$ value $(\mathrm{P}<0.05)$

Pearson correlations between the relative expression level of lncRNA AFAP1-AS1 with clinical and biochemical parameters among case group showed that the relative expression level of lncRNA AFAP1-AS 1 was positively correlated with PT, AST, ALT, direct bilirubin, and alpha-fetoprotein. On the contrary, lncRNA AFAP1-AS1 expression level was negatively correlated with, albumin, hemoglobin, and platelet $(\mathrm{P}<0.001 *)$ (Table 4$)$. 
Table (4): Pearson correlation of the relative expression level of AFAP1-AS1with clinical, anthropometric, and biochemical characteristics in HCC group

\begin{tabular}{|l|c|c|}
\hline \multirow{2}{*}{ Variables } & \multicolumn{2}{|c|}{ HCC $(\mathbf{N}=\mathbf{1 2})$} \\
\cline { 2 - 3 } & $\mathbf{r}$ & $\mathbf{p}$ \\
\hline INR & 0.089 & 0.377 \\
\hline Prothrombin time & 0.363 & $<0.001^{*}$ \\
\hline Albumin & -0.610 & $<0.001^{*}$ \\
\hline AST & 0.422 & $<0.001^{*}$ \\
\hline ALT & 0.840 & $<0.001^{*}$ \\
\hline Total bilirubin & 0.079 & 0.653 \\
\hline Direct bilirubin & 0.587 & $<0.001^{*}$ \\
\hline WBC count & 0.266 & 0.123 \\
\hline Hemoglobin & -0.497 & $<0.001^{*}$ \\
\hline Platelet & -0.852 & $<0.001^{*}$ \\
\hline Alpha fetoprotein & 0.714 & $<0.001^{*}$ \\
\hline
\end{tabular}

Linear regression analysis in the HCC group revealed that alpha-fetoprotein and ALT were the main predictors of the relative expression levels of AFAP1-AS1 among other clinical and laboratory biomarkers $(\mathrm{P}<0.001 *)$ as shown in table (5).

Table (5): Linear regression analyses to test the influence of the main independent variables against the relative expression level of AFAP1-AS1with (dependent variable) in HCC group.

\begin{tabular}{|l|c|c|c|c|c|c|c|}
\hline \multirow{2}{*}{ Model } & \multicolumn{2}{|c|}{$\begin{array}{l}\text { Unstandardized } \\
\text { Coefficients }\end{array}$} & $\begin{array}{l}\text { Standardized } \\
\text { Coefficients }\end{array}$ & \multirow{2}{*}{ t } & \multirow{2}{*}{ P value } & \multicolumn{2}{|c|}{ 95\% C.I. } \\
\cline { 2 - 9 } & B & S.E. & Beta & & & $\begin{array}{l}\text { Lower } \\
\text { Bound }\end{array}$ & $\begin{array}{l}\text { Upper } \\
\text { Bound }\end{array}$ \\
\hline Constant & 6.785 & 1.646 & & 4.452 & $<0.001^{*}$ & 3.777 & 9.993 \\
\hline Alpha fetoprotein & 0.101 & 0.036 & 0.229 & 2.755 & $<0.001^{*}$ & 0.028 & 0.173 \\
\hline GGT & 0.011 & 0.006 & 0.075 & 1.817 & 0.075 & -0.001 & 0.023 \\
\hline ALT & 0.007 & 0.002 & 0.496 & 4.350 & $<0.001^{*}$ & 0.004 & 0.010 \\
\hline
\end{tabular}

The diagnostic performance of the relative expression level of lncRNA AFAP1-AS1 in discriminating between CHC and control group.

The power of the relative expression levels of lncRNA AFAP1-AS1 to diagnose CHC among studied subjects was evaluated using ROC analysis. The AUC was $0.849(95 \% \mathrm{CI}=0.758-0.940)$ with sensitivity $=85 \%$, specificity $=75 \%$, and the cutoff values of 3.4 (Fig. 1). 


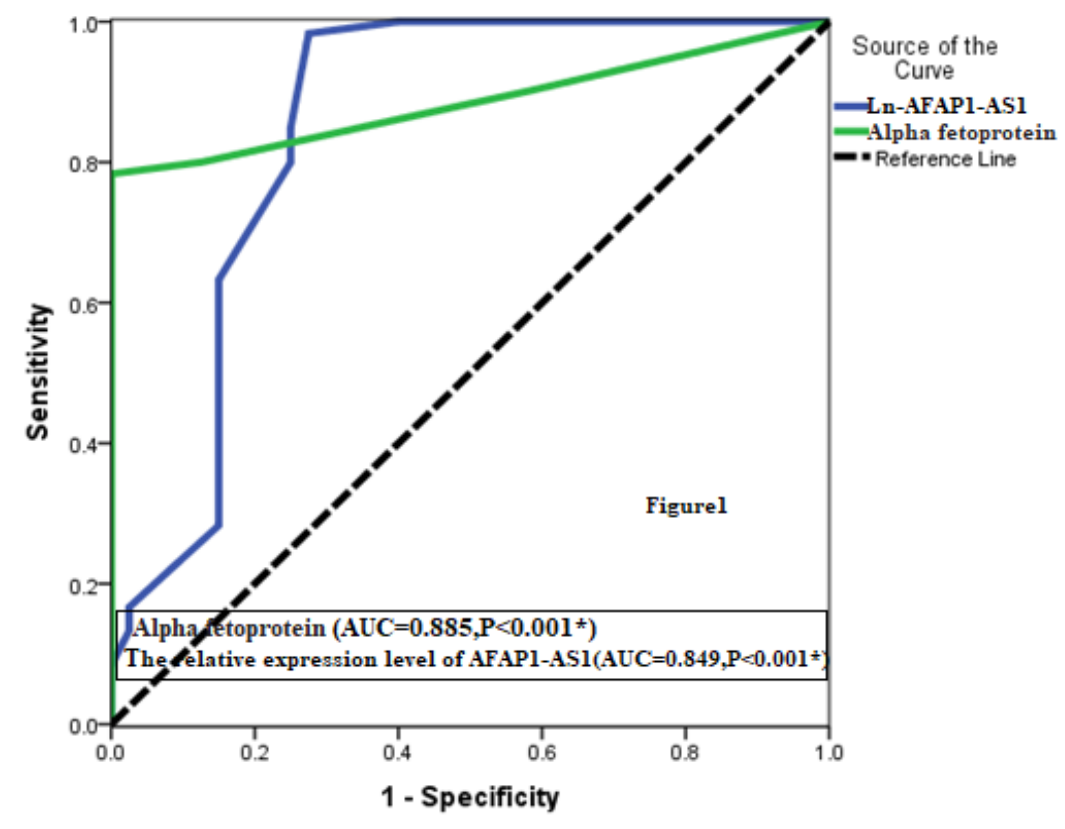

Fig. (1): Receiver operating characteristic curve of the relative expression levels of 1 lncRNA AFAP1-AS1 for diagnosis of CHC.

The diagnostic performance of the relative expression level of lncRNA AFAP1-AS1 in discriminating between cirrhosis and CHC group. The power of the relative expression levels of lncRNA AFAP1-AS1 to diagnose CHC among studied subjects was evaluated using ROC analysis. The AUC was $0.780(95 \% \mathrm{CI}=0.657-0.903)$ with sensitivity $=70.4$ $\%$, specificity $=73.7 \%$, and the cutoff value of 4.35 (Fig. 2).

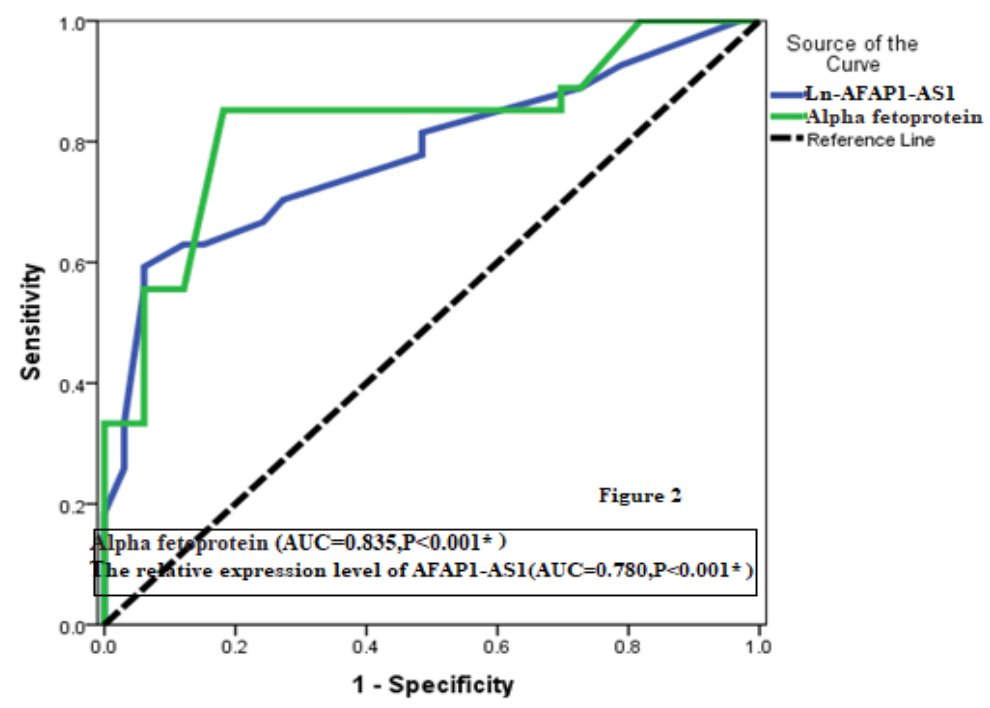

Fig. (2): Receiver operating characteristic curve of the relative expression levels of lncRNA-PVT1 to differentiate cirrhosis from CHC 
The diagnostic performance of the relative expression level of lncRNA AFAP1-AS1 in discriminating between HCC and cirrhosis. The power of the relative expression levels of lncRNA AFAP1-AS1 to diagnose CHC among studied subjects was evaluated using ROC analysis. The AUC was $0.816(95 \% \mathrm{CI}=0.709-0.924)$ with sensitivity $=83.3 \%$, specificity $=94 \%$, and the cutoff value of 104 (Fig. 3).

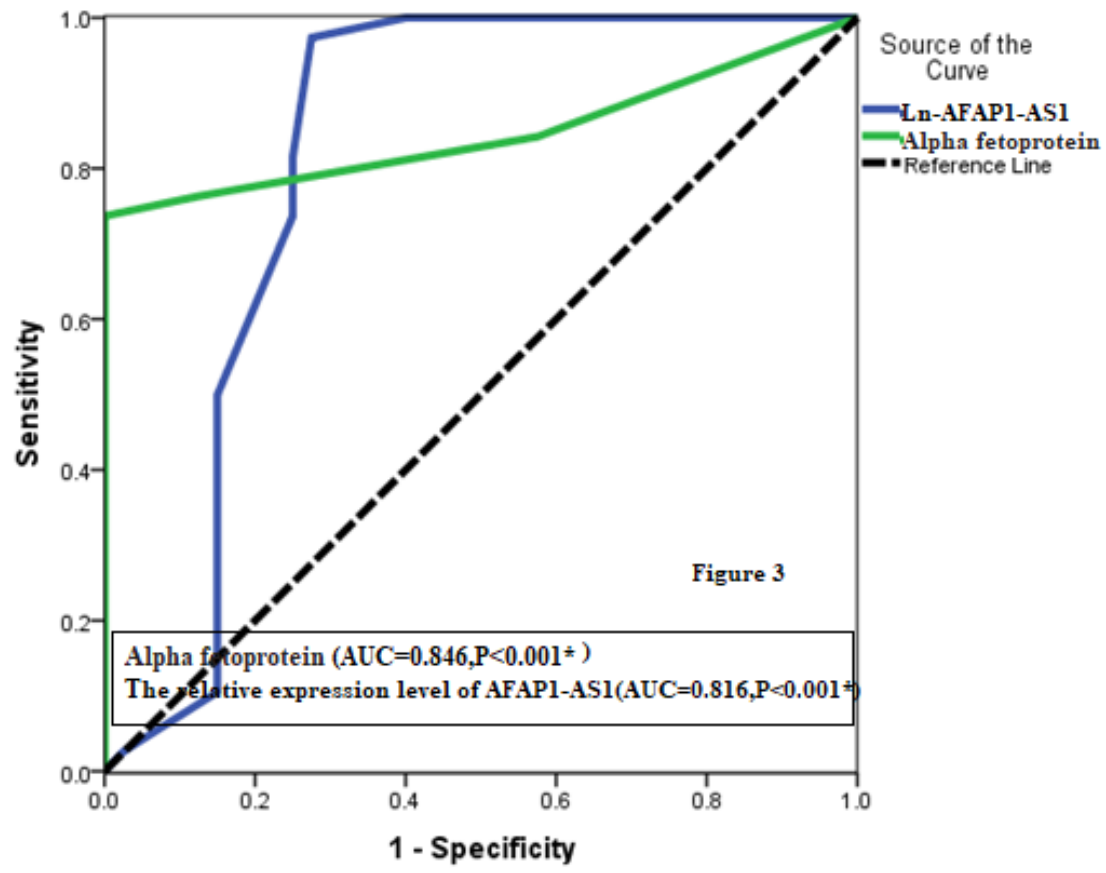

Fig. (3): Receiver operating characteristic curve of the relative expression levels of lncRNA-PVT1 to differentiate HCC from cirrhosis

\section{DISCUSSION}

$\mathrm{HCV}$ is the most prevalent cause of chronic liver disease, at present; the main causes of $\mathrm{HCC}$ are viral infections such as hepatitis B virus (HBV) and $\mathrm{HCV}$ and alcohol abuse. However, obesity and the metabolic syndrome epidemic that is occurring in Western countries is leading to a significant increase in HCC secondary to non-alcoholic fatty liver disease (NAFLD). Increasing evidence was emerging, emphasizing how the development of $\mathrm{HCC}$ is a complex and multifactorial process. The knowledge of the molecular mechanisms involved is important for the understanding of the basic factors of the development of hepatocarcinogenesis and of possible therapeutic approaches ${ }^{(\mathbf{1})}$.

Accumulating evidence supports the fact that deregulated lncRNA expression may contribute to cellular proliferation and invasion through several mRNA and proteins of growth markers and invasion markers. Previous studies showed that AFAP1-AS1 is upregulated in esophageal adenocarcinoma, pancreatic ductal adenocarcinoma, lung cancer and nasopharyngeal carcinoma ${ }^{(10-13)}$.
To the best of our knowledge, it is the first study to examine the expression levels of lncRNA AFAP1-AS1 in Egyptian patients with $\mathrm{HCV}$ and to assess its relations with clinicopathological features of HCV-related HCC. According to the current case-control study, among 60 Egyptian patients with $\mathrm{CHC}, 15$ patients had cirrhosis and 12 patients had $\mathrm{HCC}$, and the mean age of the $\mathrm{HCC}$ group was $60.2 \pm 4.8$ years and there was a significantly higher prevalence of patients with diabetes mellitus compared to other studied groups. In the current study, we adjusted BMI to avoid the influence of obesity on our findings.

These results are in accordance with the results conducted by Cancer European Organization ${ }^{(14)}$ and Lok $\boldsymbol{e t}$ al. ${ }^{(15)}$ as they reported that the prevalence of patients with HCC among HCV-related liver cirrhosis patients was about 7\%. These results agree with Davis $\boldsymbol{e t}$ al. (16), who observed that the incidence of HCC is significantly higher among elderly patients (> 60 years). Similar findings were detected by Brun et al. ${ }^{(17)}$ they observed that $\mathrm{HCV}$ is a major risk factor associated with hepatocellular carcinoma in patients with cirrhosis. The reason for this finding could be that the progression of fibrosis is related to the duration of the disease ${ }^{(17,18)}$. Other 
mechanisms explained the cause of the higher prevalence of HCC in HCV related cirrhosis suggested by Elkrief $\boldsymbol{e t}$ al. ${ }^{(19)}$ as they enumerated other factors such as diabetes mellitus, metabolic syndrome, fatty liver disease, and obesity.

Many researchers have demonstrated that $\mathrm{HCV}$ can cause alterations in the glucose and lipid metabolism and stimulate the activation of insulin-like growth factor, which in turn is responsible for the activation of hepatic stellate cells and subsequent fibrosis ${ }^{(20-22)}$. Although the pathogenesis of HCV-related HCC is mostly due to the development of cirrhosis and cell regeneration mechanisms, different alterations in gene expression and signal transduction pathways involved in cell proliferation and the neoplastic transformation of hepatocytes have been described in chronic HCV infection ${ }^{(23)}$.

The most important findings of the present study were that the relative expression level of circulatory lncRNA AFAP1-AS1 was upregulated in CHC groups. Furthermore, there was a significant difference between CHC groups' regarding the relative expression level of circulatory lncRNA AFAP1-AS1. The highest relative expression level was in HCC patients compared to cirrhosis and $\mathrm{CHC}$. These results are in accordance with Lu et al. ${ }^{(23)}$ results who investigated the expression of AFAP1-AS1 in HCC and observed that AFAP1-AS1 was considerably increased in HCC tissues compared to matched normal tissues.

The current study showed a significant positive correlation between lncRNA AFAP1-AS1 relative expression level and PT, AST, ALT, direct bilirubin, and alpha-fetoprotein. On the opposite, lncRNA AFAP1-AS expression level was negatively correlated with, albumin, hemoglobin, and platelet. Remarkably, alpha-fetoprotein and ALT were the main predictors of the relative expression levels of AFAP1-AS1 among other clinical and laboratory biomarkers.

According to our results, about $83.3 \%$ of HCC patients were in stage III/IV, and $83.3 \%$ of patients had tumor size $>5 \mathrm{~cm}$. Interestingly, there were statistically significant differences between HCC patients regarding Child-Pugh grade, portal vein thrombosis, and number of tumor lesions. This is consistent with many recent studies conducted in malignant disease; they found that the high AFAP1-AS1 expression in malignant tissue is associated with lymph node metastasis, perineural invasion, and poor survival ${ }^{(10-13)}$. Similar to our results, Lu et al. ${ }^{(24)}$ results confirmed that AFAP1-AS1 is remarkably upregulated in HCC tissues and is significantly correlated with the malignancy status and poor prognosis. Furthermore, AFAP1-AS1 silencing suppressed cell proliferation and induced cell apoptosis at least partly through regulating proliferation- and apoptosis-related genes. Accumulating evidence reported that the expression level of AFAP1-AS1 induces cellular proliferation and invasion and inhibits apoptosis by the upregulation of the RhoA/Rac2 pathway in the cell cycle, MMP-9, and PCNA protein levels, and apoptotic indices cyclinD1 along with the downregulation of $\mathrm{Bax}^{(25)}$.

For further evaluation of our results, we analyzed our results by ROC curve. The power of the relative expression levels of lncRNA AFAP1-AS1 to diagnose $\mathrm{CHC}$ among studied subjects had sensitivity $=85 \%$, specificity $=75 \%$. In addition, the power of the relative expression levels of lncRNA AFAP1-AS1 to diagnose cirrhosis had sensitivity $=70.4 \%$, specificity $=73.7$. Interestingly, the power of the relative expression levels of lncRNA AFAP1-AS1 to diagnose HCC had sensitivity = $83.3 \%$, specificity $=94 \%$.

\section{CONCLUSION}

The results of the current study showed that relative expression level lncRNA AFAP1-AS1 was upregulated in CHC patients in particular patients with cirrhosis and HCC. Thus, circulatory lncRNA AFAP1-AS1 may be able to serve as a promising non-invasive diagnostic marker for cirrhosis and HCC.

\section{REFERENCES}

1. Collaboration Global Burden of Disease Liver Cancer (2017): The burden of primary liver cancer and underlying etiologies from 1990 to 2015 at the global, regional, and national level: results from the Global Burden of Disease Study 2015. JAMA Oncol., 3: 1683-91.

2. Zoheiry M, Hasan S, El-Ahwany E et al. (2015): Serum markers of epithelial-mesenchymal transition as predictors of $\mathrm{HCV}$-induced liver fibrosis, cirrhosis, and hepatocellular carcinoma. Electron Physician, 7 (8): 1626.

3. Fattovich G, Stroffolini T, Zagni I et al. (2004): Hepatocellular carcinoma in cirrhosis: incidence and risk factors. Gastroenterology, 127: S35-50.

4. Hou J, Meng F, Chan L et al. (2016): Circulating plasma MicroRNAs as diagnostic markers for NSCLC. Front Genet., 7: 193-196.

5. Li G, Zhang H, Wan $X$ et al. (2014): Long noncoding RNA plays a key role in metastasis and prognosis of hepatocellular carcinoma. Biomed Res Int., 2014: 780-785.

6. Parasramka M, Maji S, Matsuda A et al. (2016): Long non-coding RNAs as novel targets for therapy in hepatocellular carcinoma. Pharmacol Ther., 161: 67-78.

7. Lu X, Zhou C, Li R et al. (2016): Critical role for the long non-coding RNA AFAP1-AS1 in the proliferation and metastasis of hepatocellular carcinoma. Tumour Biol., 37: 9699-9707.

8. Liovet J, Br U, Bruix J (1999): Prognosis of hepatocellular carcinoma: The BCLC staging classification. Semin Liver Dis., 19: 329-38. 
9. Pugh R, Murray-Lyon I, Dawson J et al. (1973): Transection of the oesophagus for bleeding oesophageal varices. Br. J. Surg., 60: 646-9.

10. Wu W, Bhagat T, Yang X et al. (2013): Hypomethylation of noncoding DNA regions and overexpression of the long noncoding RNA, AFAP1-AS1, in Barrett's esophagus and esophageal adenocarcinoma. Gastroenterology, 144: 95666.

11. Zeng Z, Bo H, Gong $Z$ et al. (2015): AFAP1-AS1, a long noncoding RNA upregulated in lung cancer and promotes invasion and metastasis. Tumour Biol., 37: 729-737.

12. Ye Y, Chen J, Zhou Y et al. (2015): High expression of AFAP1-AS1 is associated with poor survival and shortterm recurrence in pancreatic ductal adenocarcinoma. J Transl Med., 13: 137-141.

13. Bo H, Gong $Z$, Zhang $W$ et al. (2015): Upregulated long non-coding RNA AFAP1-AS1 expression is associated with progression and poor prognosis of nasopharyngeal carcinoma. Oncotarget., 6: 20404-18.

14. Cancer European Organization for Research and Treatment of; European Association for the Study of the Liver (2012): EASL-EORTC clinical practice guidelines: management of hepatocellular carcinoma. J Hepatol., 56: 908-43.

15. Lok A, Seeff L, Morgan T et al. (2009): HALT-C Trial Group. Incidence of hepatocellular carcinoma and associated risk factors in hepatitis C-related advancedliver disease. Gastroenterology, 136: 138-48.

16. Davis G, Alter M, El-Serag $\mathrm{H}$ et al. (2010): Aging of hepatitis $\mathrm{C}$ virus (HCV)-infected persons in the United States: a multiple cohort model of $\mathrm{HCV}$ prevalence and disease progression. Gastroenterology, 138: 513-21.

17. Bruno S, Crosignani A, Maisonneuve $P$ et al. (2007): Hepatitis $\mathrm{C}$ virus genotype $1 \mathrm{~b}$ as a major risk factor associated with hepatocellular carcinoma in patients with cirrhosis: a seventeen-year prospective cohort study. Hepatology, 46: 1350-6.

18. Rinaldi L, Nascimbeni F, Giordano M et al. (2017): Clinical features and natural history of cryptogenic cirrhosis compared to hepatitis $\mathrm{C}$ virus-related cirrhosis. World $\mathrm{J}$ Gastroenterol., 23: 1458-68.

19. Elkrief L, Chouinard $P$, Bendersky $N$ et al. (2014): Diabetes mellitus is an independent prognostic factor for major liver-related outcomes in patients with cirrhosis and chronic hepatitis C. Hepatology, 60: 823-31.

20. Tovar V, Alsinet C, Villanueva A et al. (2010): IGF activation in a molecular subclass of hepatocellular carcinoma and pre-clinical efficacy of IGF-1R blockage. J Hepatol., 52: 550-9.

21. Desbois A, Cacoub $\mathbf{P}$ (2017): Diabetes mellitus, insulin resistance and hepatitis $\mathbf{C}$ virus infection: a contemporary review. World J Gastroenterol., 23:1697-1711.

22. Irshad M, Gupta P, Irshad K (2017): Molecular basis of hepatocellular carcinoma induced by hepatitis $\mathrm{C}$ virus infection. World J Hepatol., 9: 1305-14.

23. Mesri E, Feitelson M, Munger K (2014): Human viral oncogenesis: a cancer hallmarks analysis. Cell Host Microbe, 15: 266-82.

24. Lu X, Zhou C, Li R et al. (2016): Critical role for the long non-coding RNA AFAP1-AS1 in the proliferation and metastasis of hepatocellular carcinoma. Tumour Biol., 37: 9699-9707.

25. Zhang J, Weng M, Song F et al. (2016): Long noncoding RNA AFAP1-AS1 indicates a poor prognosis of hepatocellular carcinoma and promotes cell proliferation and invasion via upregulation of the RhoA/Rac2 signaling. Int J Oncol., 48: 1590-159. 